\title{
Geste instrumental et transmission musicale
}

\section{Laurent Blum}

\section{(2) OpenEdition}

\section{Journals}

Édition électronique

URL : http://journals.openedition.org/ethnomusicologie/174

ISSN : 2235-7688

\section{Éditeur}

ADEM - Ateliers d'ethnomusicologie

Édition imprimée

Date de publication : 1 décembre 2001

Pagination : 237-248

ISBN : 2-8257-07-61-9

ISSN : 1662-372X

\section{Référence électronique}

Laurent Blum, «Geste instrumental et transmission musicale», Cahiers d'ethnomusicologie [En ligne],

14 | 2001, mis en ligne le 10 janvier 2012, consulté le 10 décembre 2020. URL : http://

journals.openedition.org/ethnomusicologie/174

Ce document a été généré automatiquement le 10 décembre 2020.

Tous droits réservés 


\title{
Geste instrumental et transmission musicale
}

\author{
Laurent Blum
}

\section{Définitions}

1 En pénétrant le mot «geste » par son étymologie, on s'aperçoit de l'existence de deux significations distinctes.

2 La première signification, aujourd'hui quelque peu oubliée, se réfère à la geste, la chanson de geste qui conte au Moyen Age les exploits d'un héros. Elle met en avant les événements marquants de l'histoire d'un individu en tentant de montrer que l'ensemble de ces gestes (l'origine de la geste vient du pluriel gesta) forment une épopée. Le destin et les particularités du caractère du héros permettent l'avènement d'une légende, la création d'une morale profane pour les générations à venir. Ce destin repose sur une vision éminemment diachronique de la vie du héros : c'est par la multitude des faits intervenus à un moment donné que le narrateur fait naître une geste.

3 La deuxième signification, plus proche de nous, évoque le mouvement d'un corps humain. Cependant, tous les mouvements humains ne sont pas des gestes. La particularité du geste s'appuie sur la force d'intention de celui qui anime son corps. La marche qui permet à un être humain de se déplacer, de transporter son corps d'un endroit à un autre, appartient plus généralement au domaine du mouvement. Par contre, le salut d'un être humain vers un ou plusieurs autre(s) est bien un geste : il suffit d'un signe de la main, même à grande distance de celui qui le perçoit, pour reconnaître immédiatement la volonté de communication de celui qui le produit. La description minutieuse de la façon dont la main de l'émetteur a bougé, s'est élevée du niveau de la hanche vers la hauteur de sa poitrine, la décomposition des mouvements du poignet s'épuisent à rendre compte de la spécificité du salut. Le signe ou symbole récapitule de façon globale et instantanée l'intention du geste.

4 Le croisement de ces deux expressions - la geste et le geste - permet de rendre compte de la problématique de la transmission musicale. Une expérience d'enseignant évoquée par 
Maurice Martenot (1970: 148), convainc que la force du geste se saisit dans l'instant même. Pour obtenir de la part d'un élève qu'il émette un « Hep! » sonore, l'enseignant a le choix entre, d'une part, la description des mouvements à accomplir et du résultat sonore escompté et, d'autre part, une mise en situation réaliste. Dans le premier cas, l'enseignant décrit la respiration abdominale et le timbre de voix riche en harmoniques qu'il souhaiterait que l'élève émette lors de son «hep!». Dans le deuxième cas, il demande à l'élève d'imaginer qu'il se trouve au milieu d'une foule et qu'il reconnaît soudain un ami qu'il n'a pas vu depuis longtemps. Inconscient du regard qui pèse sur lui, celui-ci s'éloigne à grands pas. Alors qu'il est sur le point de disparaître de sa vue, l'élève doit crier le « hep ! » qui pourrait avertir cet ami de sa présence.

5 On devine que le meilleur moyen pour obtenir ce «hep!" sonore correspond à la deuxième solution. Pour reconstituer un geste, il faut créer la situation qui met l'élève devant un défi. La reproduction du geste, dans sa dimension d'apprentissage où l'élève s'exerce, découvre et corrige, sera de l'ordre de l'analyse diachronique : décomposition des muscles sollicités, discrimination entre le geste et la trace sonore. Nous quittons le domaine de la "performance » pure, c'est-à-dire de la prestation ou encore de la "monstration». Le déroulement pédagogique dans le domaine artistique se bâtit par imprégnation et réflexion. L'artiste professionnel qui est son propre professeur, se trouve confronté à ce temps double, temps engourdi de la préparation s'opposant au temps brutal du spectacle.

6 Le prochain chapitre reconstituera les événements marquants de l'épopée des instruments de musique. Entre leur origine utilitaire, appartenant à la vie domestique et, à l'opposé, leur caractère parfois sacré, les instruments se manifestent dans tous les aspects de la vie sociale traditionnelle.

\section{L'épopée des instruments de musique}

7 Nous retiendrons la classification habituelle des instruments de musique pour faire émerger une histoire de la gestualité dans chaque famille d'instruments. Dans chacune de ces familles existent des instruments hybrides; certains pourront être évoqués pour mieux illustrer ou tempérer le propos mais leur multiplicité nous interdit évidemment de présenter de façon exhaustive le kaléidoscope des gestes liés à cette famille.

\section{Les instruments à vent}

8 Un instrument grec, l'aulos, traduit à tort dans tous les textes de langue française par flûte, peut être considéré comme l'emblème de cette famille. Cet instrument constitué probablement d'une anche simple, fut l'un des instruments les plus joués de la civilisation hellénique, et pourtant, dès le $\mathrm{II}^{\mathrm{e}}$ siècle après Jésus-Christ, les témoignages de son existence se font très rares. Que s'est-il passé ?

9 La caractéristique des instruments à vent, outre le fait que le son est produit habituellement par le souffle humain, se manifeste par une relation charnelle avec l'instrumentiste, que cette relation soit présentée en filigrane ou de manière abrupte. Il suffit de penser au rôle que joue la bouche de l'instrumentiste pour s'en convaincre. Ingérant une extrémité dans le cas des instruments à anche, collant leur bouche contre l'embouchure dans le cas des flûtes, les instrumentistes à vent fusionnent de manière 
physique avec leur instrument. Regardons le trompettiste créant avec ses muqueuses les « flattements » responsables des sons émis par l'instrument.

Battements d'anche à l'intérieur de la cavité buccale, vibrations lippales créent simultanément chez l'auditeur un sentiment de dégoût face à l'aspect malpropre de cette musique, et une forme d'attirance pour la chaleur des sonorités de ces instruments. Des témoignages sur l'aulos nous montre l'ambivalence dans laquelle les instruments à vent se situent: lorsque l'aulète joue, il se tient «les mâchoires gonflées, élevées, les yeux exorbités, injectés de sang ou alors le visage calme, malhonnête, ignorant l'expiration » (Pollux 1900 : 68).

11 L'instrumentiste, par les convulsions de son visage, crée une certaine frayeur au sein du public et, si ses traits ne sont pas convulsés, il faut alors en avoir une plus grande méfiance encore, car son air impassible dissimule certainement des desseins inavouables. C'est ainsi que l'épopée des instruments à vent est plutôt la double histoire d'une fascination et d'une malédiction. Banni par les Grecs en raison de son origine asiatique, puis adopté par eux, à tel point qu'il devient l'instrument obligé des orgies dionysiaques, l'aulos est à nouveau écarté des sphères musicales au moment de la naissance du christianisme. Les instruments à anche ne réapparaîtront progressivement qu'à la fin du Moyen Age.

Parallèlement à la caractéristique charnelle de l'instrument à vent, la notion de dépense, d'excès, détermine le geste du joueur de cette famille. La mise en vibration d'une colonne d'air, ensemble des particules gazeuses en mouvement à l'intérieur du tuyau de l'instrument à vent, nécessite une pression abdominale très importante de la part du joueur. Elle ne restitue qu'une infime partie de son énergie en énergie sonore. Ainsi, entre l'effort fourni par le joueur et ce que le public peut entendre, la perte d'énergie est considérable. La réflexion de Pollux réapparait avec une pertinence accrue : la force du son « venté » tient dans son caractère double ; le geste se révèle, de façon énigmatique, alternativement convulsif et serein.

Certains instruments comme les flûtes à bec occidentales ou le ney iranien se démarquent $\mathrm{du}$ caractère fougueux des instruments à vent. Leur son éolien a un charme qui fait oublier les aspects charnels de leur production sonore. Cette distinction n'a pas eu l'heur de plaire aux compositeurs de la période classique et romantique parce qu'il leur manquait précisément la puissance sonore. Une puissance ne représente pas seulement le volume en décibels d'un son, mais bien une répartition spécifique des partiels qui provoquent un effet de saturation chez l'auditeur. Le résultat musical, tant est que nous pouvons décrire un résultat musical, donne l'impression à l'auditeur qu'il n'entend qu'une partie du son, que ni les caractéristiques de son oreille, ni les capacités acoustiques de l'instrument ne sont en mesure de restituer entièrement.

La particularité des sons des trompes, des cors ou des mey (instruments à anche double et à tuyau cylindrique, qui créent une perception indirecte comme en écho) joue sur cette dualité présence-absence, sur cette ambivalence de puissance détournée.

\section{Les instruments à cordes}

15 Cette famille recouvre aussi une multitude d'instruments variés. Nous éviterons toute nomenclature fastidieuse et tenterons de cerner les caractéristiques essentielles du geste instrumental. 
16 A l'ambivalence et à l'excès des instruments à vent, la famille des instruments à cordes répond par une relation beaucoup plus en symbiose avec le monde environnant. La harpe éolienne, instrument à cordes très sensible, illustrera notre propos: l'intervention humaine pour la production sonore de cet instrument est ici minimale. Il suffit que l'instrument soit judicieusement orienté par rapport au vent pour que ses cordes vibrent. A l'instar du feuillage des arbres, la harpe éolienne semble émettre des sons directement issus du monde céleste. L'instrumentiste à cordes se comportera comme un médiateur entre une nature en soi généreuse et l'oreille humaine. Le grattage ou le frottement de la corde par l'instrumentiste permet à la corde de produire le son fondamental. La facture des instruments à cordes se mettra progressivement au service du geste humain en créant des caisses de résonance adaptées à l'enlacement. Le violoncelle, de par sa dimension et sa tenue, offre un exemple typique de l'analogie entre le corps humain et la facture instrumentale: entre le joueur dont le travail quotidien s'articule autour des différentes manières d'embrasser son instrument et la facture résultant d'une transformation perpétuelle des galbes et des creux de l'instrument pour mieux accueillir le corps de l'instrumentiste, le geste musical se fonde dans une recherche persistante de l'harmonie. Schafer (1979) présente ainsi les deux tendances de l'histoire de la musique : la tendance dionysiaque représentée par les instruments à vent dans laquelle l'homme s'arroge le rôle de démiurge et la tendance apollinienne, dont la lyre est l'instrument roi, qui s'appuie sur la recherche du beau, de l'harmonieux, du proportionné. Cette école apollinienne se prolonge par la démonstration d'Aristote à propos de la décomposition du son en harmoniques : en divisant la longueur d'une corde par 2, 3 ou 5, nous obtenons respectivement l'octave, la quinte et la tierce supérieures. Ainsi sont démontrées l'harmonie de l'univers et les correspondances entre les mondes visuel et auditif.

17 L'enlacement de l'instrument s'accompagne d'une pédagogie du centre de gravité: étreindre et faire vivre un morceau de bois tendu de quelques cordes implique une forte connaissance de l'équilibre de celui-ci et une étude des points de contact entre eux. Suspendu telle la harpe éolienne mais enlacé à la manière d'un être cher, l'instrument à cordes dévoile sa richesse sonore par effleurement. Le geste est ici projection. L'esquisse est la caractéristique du mouvement de l'instrumentiste à cordes. Toucher délicatement et faire de son corps à l'instar du corps de l'instrument le réceptacle du son est le nœud gestuel de cette famille. Créer un espace d'intimité, suggérer l'idée musicale appartient aux qualités de l'instrumentiste à cordes.

Une distinction entre instruments de plein air et instruments de chambre tempère évidemment cette proposition. Parmi ces derniers, la vielle à roue, très prisée dans les salons du XVIII ${ }^{\mathrm{e}}$ siècle, donne à entendre simultanément les bourdons et la ligne mélodique. Sa sonorité aigre ne suggère en aucun cas un instrument d'intimité.

La famille des cordes frottées utilisées dans les orchestres occidentaux mérite une mention particulière. Les archets des violons plus tendus et les bois de construction des caisses de résonance plus tendres ont permis aux instrumentistes de produire des transitoires d'attaque plus francs et des sons plus perçants. En multipliant le nombre des instruments à cordes dans les orchestres symphoniques du XIX ${ }^{e}$ siècle, en incluant dans les partitions des répétitions stretto en doubles-croches,les compositeurs ont provoqué dans le rang des violons une floraison de sonorités éclatantes dont les violes étaient dépourvues. La technique française du violon telle qu'elle a été élaborée sous la direction de Lully peut s'enorgueillir d'une précision quasi militaire du coup d'archet. Sans 
atteindre l'agressivité des instruments à vent, ces techniques ont permis l'épaississement des timbres et le renforcement des dynamiques.

\section{Les instruments de percussion} les milieux de l'enseignement musical spécialisé. La pédagogie percussive serait une pédagogie binaire. Cette affirmation se réfere à la percussion avec une baguette dans chaque main dans le sens le plus étroit : apprentissage des roulements et autres rythmes obstinés sur membranophones. Le paysage gestuel qu'offre cette famille est pourtant immense : la multiplicité des instruments auquel le percussionniste fait face pendant l'interprétation d'une œuvre, l'alternance entre plusieurs jeux de baguettes nécessite de la part du joueur une maîtrise de l'espace, une connaissance intime de la place et du trajet qu'est susceptible de parcourir en un temps donné l'objet frappant jusqu’à l'objet frappé et réciproquement. Le joueur exécute un véritable ballet (ballein en grec signifie jeter) pour anticiper et maîtriser les événements sonores. Si nous examinons le vaste monde des percussions digitales utilisées dans toutes les musiques traditionnelles, la dualité main gauche/main droite apparaît une donnée extrêmement pauvre face à la multiplicité des frappes possibles des différentes parties de la main sur les différentes parties de l'instrument.

22 La deuxième assertion qui mérite d'être mise en question concerne l'approche de la percussion comme un renforcement d'un rythme pré-écrit ou pré-formalisé d'une œuvre musicale. Il est vrai que l'histoire musicale de l'Occident, lors de l'avènement de la polyphonie, a relégué le rôle de la percussion à celui d'un cadre temporel, sorte de guide favorisant la synchronisation du jeu des instrumentistes. Aussi les percussions occidentales se sont-elles davantage développées dans les musiques populaires dansées. Là encore, elles représentent un appui rythmique auquel les danseurs se réfèrent. Les mouvements des danseurs comme les gestes des musiciens sont synchronisés grâce à la pulsation régulièrement frappée ou percutée.

Philippe Vigreux (1999: 15-27) présente, en introduction de sa méthode de derbouka (membranophone utilisé dans la majorité des pays arabes), le rôle traditionnel de la percussion : le rythme y apparaît comme un « informateur de mélodie ». Si le rythme est aussi tributaire d'une périodicité, cette période s'appuie d'abord sur la prosodie, donc sur des phrases qui peuvent s'enchaîner souplement les unes aux autres. L'art de l'ornementation rythmique crée sa propre dynamique, s'éloigne pour revenir régulièrement sur les traces de la mélodie. Il est alors difficile de souscrire à la distinction que propose Pierre Boulez (1987) entre musique striée et musique lisse. Envisagée dans le cadre de la musique occidentale, elle peut coïncider avec la distinction entre la musique savante contemporaine et les « autres » musiques occidentales, tributaires de la mesure. Si ces dernières sont marquées par une pulsation régulière et même si l'interprétation de ces œuvres produit une agogique à chaque fois différente, la musique contemporaine s'efforce de quitter ce temps scandé en créant une succession d'événements sonores, ne 
dépendant plus d'une forme de synchronisation rythmée pulsée, mais fonctionnant comme des signaux de départ pour la séquence suivante.

Il a été beaucoup écrit sur les polyrythmie africaines. Autant sur le plan abstrait, l'Occidental peut comprendre lorsqu'il entend une superposition de cycles autonomes, autant sur le plan concret, son oreille ne peut saisir simultanément le déroulement de ces cycles. Pourtant le percussionniste africain interprète le cycle dont il est responsable, cycle parfois constitué de périodes très longues, et, simultanément, compose avec les cycles interprétés par les autres percussionnistes, de telle manière que l'ensemble crée une architecture typique. Cette dernière-ci a déjà été signalée par Jones $(1959: 210)$ qui estime qu'elle se décline dans toute l'Afrique noire en une succession de douze unités de temps sous la forme suivante : $2+2+3+2+3$.

La difficulté de cerner l'épopée des instruments de musique ne réside pas seulement dans leur multiplicité et la diversité des interprétations musicales. Le geste de l'instrumentiste contribue à faire découvrir la complexité de l'instrument et la recherche parfois antinomique entre le compositeur et le luthier : si ce dernier tente de réaliser un objet capable de fournir des sons stables et homogènes sur la plus grande étendue possible, le compositeur cherche au contraire souvent les limites des capacités sonores de l'instrument.

\section{Les archétypes de la transmission instrumentale}

A partir des pistes de réflexion que nous a inspirées l'épopée des instruments de musique, nous pouvons tenter de proposer des archétypes du geste instrumental. Signalons cependant notre réserve sur la particularité de ceux-ci. Ils ne sont à mettre au compte d'un héritage universel de l'humanité que parce qu'ils sont à l'œuvre dans un lieu déterminé. François Wahl (1999 : 5) commente en ces termes la « vérité » du geste :

«De toute nécessité en un point du discours: il n'y a de vérité que locale. Mais substantiellement, ouvrant sur ce qui, du sein du discours, déborde le discours même : il n'y a de vérité qu'infinie». C'est dire encore une fois que le geste s'inscrit dans l'interprétation hic et nunc d'une œuvre : de façon paradoxale, il est universel parce qu'il est local.

\section{Transmission : technicité et imprégnation}

Trois types d'activités sont nécessaires pour qu'un groupe de musiciens, professionnels ou amateurs, puisse se produire: la fabrication de l'instrument de musique, la conceptualisation de l'œuvre et l'interprétation de celle-ci. Les musiques savantes (occidentales ou non) répondent aux différentes phases de production en créant une spécialisation dans chacune des activités; dans les musiques de tradition orale, les musiciens cumulent par contre souvent les trois fonctions. La démarche de l'interprète, et plus particulièrement celle de l'instrumentiste, se situe entre les deux extrêmes : d'une part, l'hyper-spécialisation confinant à une vision technique de la musique et, d'autre part, l'adaptation progressive à la fabrication du son dans les trois domaines de la production musicale.

29 Le musicien «traditionnel » effectue les trois phases par approximations successives et alternées. Tel l'artisan qui tient une cuillère dans sa main et, la soupesant, la déclare 
«parfaite » (Maneveau 1970 : 17), le musicien traditionnel décide - de façon collective au moment de l'interprétation qu'il est dans le vrai, que son interprétation est le reflet de son travail, de son rôle social et de son identité culturelle.

La transmission d'un savoir-faire d'un musicien à un autre interfère directement sur le geste lui-même. Pris dans la technicité de son geste, le musicien occidental se concentre sur des éléments extra-musicaux telle, dans le dernier exemple cité à propos des percussions, l'indépendance des mouvements de la main droite et de la main gauche. La fascination pour les détails - particularités de la facture de l'instrument ou découvertes de ses propres limites physiques - lui fait oublier la raison d'être de ce mouvement. Au contraire, le percussionniste africain inscrit ses gestes dans son cadre quotidien, quelles que soient leur nature et leur fonction : ainsi s'imprègne-t-il de l'environnement social constitué de repères familiers.

31 Le travail instrumental s'effectue simultanément à l'écoute des propositions musicales de ses partenaires. Nous sommes en face d'une œuvre en devenir, d'un work in progress, évidemment sans l'indétermination prônée par John Cage et ses successeurs; pour comprendre les différentes étapes de la gestation de ces œuvres pour percussions, il faudrait accompagner les musiciens durant le lent processus d'apprentissage et d'intégration dans le groupe. Il est cependant clair que cette façon de travailler présuppose une capacité d'écoute et d'anticipation sur les événements sonores dont les musiciens occidentaux sont parfois dépourvus.

32 La situation de l'informaticien, dans son rôle de créateur d'événements sonores, semble atypique. Son geste reconstitue le geste musical dans sa dimension synchronique; il ignore le long itinéraire de la naissance de l'idée musicale dans le contexte social, les différentes étapes de la fabrication de l'instrument de musique, les recherches structurelles des compositeurs et l'histoire des différentes interprétations des œuvres musicales. Sa recherche par tâtonnements s'apparente à celle du musicien traditionnel, mais démuni des racines d'un groupe ou d'une ethnie spécifique, il fait appel à son background personnel constitué de la multiplicité des sources musicales dont tout consommateur de culture dispose aujourd'hui. Il est un peu comme un bâtisseur de cathédrales gothiques disposant de pierres de taille aux dimensions et à la résistance requises, mais sans la connaissance de l'architecture et sans la motivation spirituelle qui animait ceux-ci. Ses matériaux ont une richesse potentielle extraordinaire, mais sa dépendance technologique semble l'empêcher d'édifier à partir de cette potentialité un geste musical susceptible de s'inscrire dans le temps.

33 Cette dépendance technologique n'est cependant pas la conséquence de la transmission instrumentale d'une école; elle est le fait, comme nous le verrons en conclusion de cet article, d'un emprisonnement volontaire.

\section{L'articulation ou l'appel du silence}

Avant que le timbre d'un son n'apparaisse - timbre des instruments à sons déterminés ou indéterminés sur le plan de la hauteur -, mais après que le son précédent ait disparu, il y a ce temps du silence ou de la juxtaposition, bref un moment de transition qui est traité depuis toujours avec beaucoup de soin par le musicien. Dans ces longs legato durant lesquels chaque son semble quitter à regret l'espace pour laisser place au son suivant, l'articulation s'appréhende au niveau macroscopique. La fusion des séquences sonores permet au compositeur de quitter le champ du geste instrumental individuel pour se 
consacrer à l'architecture de l'œuvre. Cette opération n'est pas bénigne : le geste musical semble prendre son envol pour des contrées plus abstraites. Déplacements de masses sonores, transformations évolutives des timbres, la musique emplit l'espace de manière continue, crée un temps lisse durant lequel tout avènement n'est qu'une résurgence d'un présent déjà là. La conception de cette musique lisse semble issue directement de la vision céleste du savant jésuite du XII ${ }^{e}$ siècle, Athanase Kircher qui évoque la harpe éolienne et son cortège de sons immanents emplissant l'univers (fig. 1).

Fig. 1 : Athanase Kircher, Musurgia universalis, 1650 (page de garde)

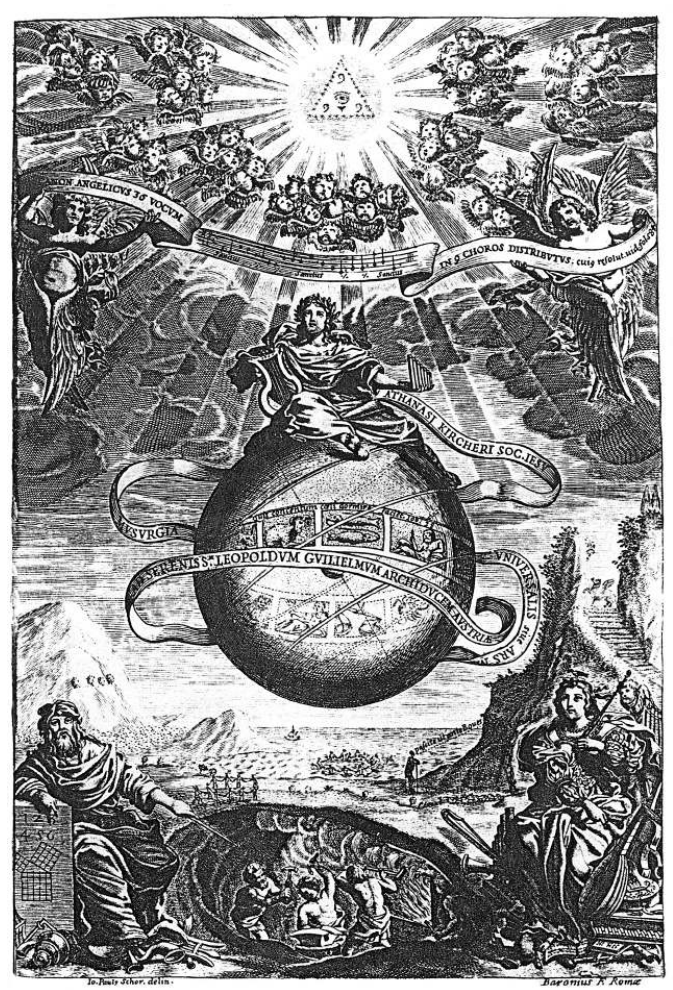

A l'opposé, le geste instrumental articule les sons. La naissance de chacun d'eux est l'objet d'une maîtrise, le résultat d'une volonté individuelle. Fort de cette responsabilité, le joueur conjuguera toutes les formes musicales pour faire advenir le timbre. L'ornementation est l'art de préparer, d'anticiper, de surprendre, de retarder. La création de ces intermèdes s'est développée dans toutes les civilisations; celles qui ont mis en avant le principe mélodique ont multiplié les ports de voix, les appoggiatures et autres mordants. On peut y déceler, en parallèle à la rhétorique, un jeu sur la feinte, une science de l'évitement afin que points d'appui et notes de passages se relaient en une joute continuelle. Entre la construction géométrique de la structure et l'assemblage des sons, l'intervention du joueur perdure.

Dimension microscopique (succession des sons) et dimension structurelle, qualité du geste et analyse de la forme, dans les deux cas, la musique est l'art d'accommoder les interstices. L'extinction d'un son fait figure d'annonce pour le prochain; pour transmettre cette caractéristique de l'aspiration au futur immédiat de l'appel musical, le pédagogue utilise toutes les images de l'offrande. Le geste de donner permet à celui qui reçoit, comme à celui qui donne, de transformer son état. Le son est lui-même le geste 
émis et perçu, le tympan de l'auditeur venant à la rencontre - en creux - de la membrane, de l'anche, de la corde de l'instrument de musique.

L'art des muses est ainsi un art physique, qui fait intervenir l'oreille interne avant même qu'un son ne soit émis. Notre cerveau est en effet capable de mettre en mouvement les cils de l'oreille interne pour les mettre en résonance avec les sons que nous imaginons, que nous anticipons. La projection auditive ou écoute active n'est donc pas seulement de l'ordre de l'imaginaire. Avant même que le geste instrumental ne soit perçu, il a déjà pris corps.

\section{Le timbre ou la perte d'identité}

Le son musical est doté d'une énergie particulière dépendant de l'attention de l'interprète et de l'auditeur. Ceux-ci doivent se comporter en état d'alerte permanente. L'ouïe est un sens en quelque sorte archaïque, qui n'a pas libéré l'homme de son emprise depuis la préhistoire. Avant d'être conquérant, prédateur pour les autres espèces, il fut d'abord gibier: ses principales défenses étaient certainement un sens auditif aiguisé qui lui permettait de localiser rapidement la nature et le lieu d'où l'attaquerait son prédateur. C'est pourquoi l'oreille humaine cherche encore dans les sons qu'elle perçoit à caractériser leur source.

Le timbre, qui, sur plan physique, se définit par la répartition des partiels sur l'échelle des fréquences, montre l'intérieur de l'enveloppe sonore. Chaque instrument de musique possède une signature "timbrique» à laquelle, pourtant, le musicien échappe constamment. Il n'est que le XIX ${ }^{\mathrm{e}}$ siècle occidental, en raison de ses instruments figés, finis, et d'une recherche exacerbée des industriels vers l'homogénéité, pour avoir voulu créer des modèles sur lesquels les compositeurs auraient créé des œuvres aux formes parfaites. L'exploration du timbre est l'apanage de la plupart des musiques modales traditionnelles et elle s'est considérablement développée dans la musique occidentale savante depuis la fin du XIX siècle. Les subtils détours et errements de ces musiques, avant d'effleurer (parfois) le son pivot, montrent paradoxalement l'importance que revêt la source. Toujours suggéré et fluctuant, le timbre caractéristique de l'instrument fonctionne surtout comme un leurre.

40 L'art créateur commence par un deuil ; c'est à l'absence que la musique s'adresse. Les artisans de la musique - luthiers, compositeurs, interprètes - sont responsables d'une création de l'évanescent. L'industrie de la lutherie et de la production de disques a la lourde tâche de transformer cet évanescent en produits de consommation. Incipit de l'énigme de l'univers, introduction de la perte de parole dans la communication, les modes de production musicale fêtent l'absence du sens, tant dans les interstices qui ne sont que moments d'attente, que dans l'écoute intérieure du timbre qui ne signe jamais sa source. L'instrumentation choisie pour chaque interprétation musicale dévoile ce sentiment de perte. Ambivalence des instruments à vent, harmonie des instruments à cordes, frappe des instruments de percussion se combinent pour créer un exode continu.

41 Pour conclure, l'informatique musicale devient le point de rencontre de différentes pratiques musicales. Le geste se trouve confronté, aujourd'hui plus que jamais, à la double gageure de l'évolution de la technologie et de l'éducation artistique.

Il existe depuis des millénaires des instruments raclés constitués de pierres gravées sur lesquelles on frottait une baguette de bois (peigne). Ces lithophones fonctionnaient avant 
la lettre comme des électrophones. Les instruments à clavier reprennent ce principe d'enregistrement, mais en abandonnent l'aspect linéaire, en transformant et en multipliant les peignes pour fabriquer des pinces (clavecin) ou des marteaux (pianoforte) mettant des cordes en vibration. La gestion du temps est devenue distributive, favorisant une polyphonie créée à partir de la configuration digitale de l'instrumentiste. Un pas sera à nouveau franchi avec les synthétiseurs, puis un autre avec les ordinateurs. La distribution spatiale laisse place alors à des générateurs de sons dans lesquels le geste ou son résultat sonore est représenté sur l'écran.

C'est à cette problématique que le musicien, a fortiori l'informaticien musical, est aujourd'hui confronté. Comment créer une forme musicale qui suggère l'alerte, qui donne à entendre la présence/absence de la source sonore? La transformation d'un geste musical en une composition d'informations sous forme binaire risque de se réduire à une dépendance stricte du geste technique. Comment une synthèse analogique des timbres peut-elle nouer un fil conducteur avec l'épopée des instruments de musique ? Comment peut-elle inventer un geste comportant l'imminence de l'événement sonore à venir? L'une des voies les plus prometteuses est celle de la composition mixte en temps réel: entre un instrumentiste traditionnel, utilisant des instruments acoustiques, et l'informaticien, gérant un programme évolutif en fonction des gestes de l'instrumentiste, la composition musicale peut à nouveau réaliser des articulations entre les séquences dans une continuité de la recherche de l'inouï.

\section{BIBLIOGRAPHIE}

BOULEZ Pierre, 1987, Penser la musique aujourd'hui. Paris : Gallimard.

BRETON Luc, 1989, A propos du tracé directeur de l'instrument à cordes. Cahiers de musiques traditionnelles 2 : « Instrumental ». Genève: Ateliers d'ethnomusicologie / Georg: 217-233.

JONES A. M., 1959 , Africa and Indonesia. The evidence of the xylophone and other musical and cultural factors. Leiden : Brill.

KIRCHER Athanase, 1650 , Musurgia Universalis I. Rome : Corbelleti.

MANEVEAU Guy, 1970, Musique et éducation. Toulouse : Edisud.

MARTENOT Maurice, 1979, Principes fondamentaux de formation musicale et leur application. Paris : Magnard.

POLLUX Julius, 1900, Onomasticon IV (IIe s. ap. J.-C.). Texte établi par Ericus Bethe. Lippe : Teubner.

SCHAFER R. Murray, 1979, Le paysage sonore. Paris : Lattès.

VIGREUX Philippe, 1999 [1985]La derbouka. Technique fondamentale et initiations aux rythmes arabes. Aix-en-Provence : Edisud.

I. WAHL François, 1999, Le geste musical mis à nu. Paris :Conférence IRCAM, 27 février 1999. $<$ www.entretemps.asso.fr/Nicolas/Samedi/Wahl.html> 


\section{RÉSUMÉS}

Le geste instrumental recouvre un domaine extrêmement large puisque les différentes disciplines musicales - analyse auditive, histoire de l'interprétation et des idées musicales, esthétique, etc. - sont directement interpellées par cette expression. Après une incursion dans la polysémie du geste et une description de l'histoire des instruments de musique, un tableau synthétique des aspects fondamentaux du geste musical lié à l'instrument de musique permet d'esquisser des pistes de réflexion sur les pratiques musicales et leur enseignement.

\section{AUTEUR}

\section{LAURENT BLUM}

Laurent BLUM, diplômé de clarinette du Conservatoire National de Région de Metz, parcourt le cursus universitaire musical jusqu'au doctorat en musicologie sous la houlette de Guy Maneveau. En 1985, il rencontre le clarinettiste Memet Ermakastar qui lui fait découvrir un jeu instrumental nouveau. Son travail de recherche l'amène à déposer un brevet sur un nouvel instrument de musique, le «modulair», instrument hybride qui permet au musicien débutant de découvrir de manière ludique la facture et la pratique des instruments à vent. Ayant enseigné la musique de chambre et dirigé l'orchestre d'harmonie à l'Université de Rennes pendant une dizaine d'années, il est appelé à la direction des dix-huit écoles de musique de Strasbourg, puis à celle du Conservatoire Populaire de Musique de Genève. 